\title{
HYDRAULIQUE
}

\section{La Solution générale du Problème de la Détermination des Dimensions Economiques Maximum d'une Conduite forcée en Métal et son Application aux Calculs pratiques.}

Par Paul P.-SANTO RINI, Ingénieur E. P. Z., Directeur de la "Sociélé Anonyme d'Etudes et d'Entreprises ", Athènes.

\section{VI. - CONDUITE D'UN SEUL DIAMETRE CONSTANT}

Il s'agit ici d'un cas qui se rencontre très souvent en pratique. En effet, si la longueur de la conduite ne justifie pas la subdivision en plusieurs tronçons de diamètres différents, le constructeur est très souvent enclin de ne prévoir qu'une conduite d'un seul diamètre constant, aussi bien dans la section amont que dans la section aval.

Une autre raison qui peut imposer une pareille conduite de diamètre unique est celle liée à la vilesse maximum de l'eau. En effet, nous avons démontré à plusieurs reprises, dans le courant de cette étude, que plus le nombre de tronçons $n$ est grand, plus petit devient le diamètre aval, plus grand devient le diamètre amont.

Or, la vitesse maximum $n$ étant fonction du diamètre aval, il arrive très souvent que même avec $n=1$, le diamètre ainsi obtenu se trouve insuffisant pour débiter le volume d'eau $Q$ dans les limites admises de la vitesse $\mathrm{U}$.

Il reste alors, abstraction faite de la répartition du débit Q sur deux ou plusieurs conduites, comme dernière ressource, l'adoption d'une conduite d'un seul diamètre constant.

Ce cas n'ayant jamais été résolu jusqu'ici, nous mentionnerons que la fonction bénéfices prend la forme:

$$
\begin{aligned}
& \mathrm{B}=\mathrm{B}_{0}-\left[\frac{\mathrm{B}_{1}}{\mathrm{D}^{5}} \int_{\mathrm{i}_{u}}^{\mathrm{I}} d l+\mathrm{B}_{2} \mathrm{D}^{2} \int_{\mathrm{i}_{u}}^{\mathrm{L}} h d l\right]- \\
& -\left[\frac{\mathrm{B}_{1}}{\mathrm{D}^{5}} \int_{0}^{l_{0}} d l+\mathrm{B}_{2} h_{0} \mathrm{D}^{2} \int_{i}^{l_{0}} d l\right]
\end{aligned}
$$

les termes $B_{0}, B_{1}$ et $B_{2}$ résumant trois constantes dont nous n'avons guère lieu de nous occuper ici, D signifiant, d'aulre part, le diamètre unique pour la section amont et aval.

En annulant la dérivée par rapport à $\mathrm{D}$, nous obtenons :

$$
\mathrm{D}=\sqrt[7]{\frac{\mathrm{TL}}{\int_{0}^{\mathrm{L}} h d l+h_{0} l_{0}}}
$$

done :

$$
\mathrm{I})=\sqrt[7]{\frac{\mathrm{TL}}{y\left(\mathrm{~L}-l_{0}\right)+h_{0} l_{0}}}
$$

où nous rappelons que $y$ est la charge statiçue du centre de gralvité de l'axe de la section aval de la conduite forcée: $h_{0}$ est lia charge statique (constante constructive) séparant la section amont de la section aval; $l_{0}$ est la longueur de laxe de la section amont de la conduite et $\mathrm{L}$ est la longueur lotale de la conduite.

On s’aperçoil que dans celle formule le diamètre est fonction de la longueur de la conduite. Cest là un résulat absolument différent de tous ceux obtenus jusqui ci. lin effet, tous les auteurs s'étant jusqu'ici occupés du problème qui nous intéresse sont unanimes à obtenir la valeur du diamètre indépendante de la longueur de la conduite. Ce résultat erroné est dû au fail qüils se bornáient toujours à considérer séparément la section aval de la section amont de la conduite. Or, il est facile de démontrer que c'est précisément la présence inévitable de la section amont qui laisse apparaitre la longueur de celte section par rapport à la longueur tolale de la conduite dans la formule exprimant la valeur du diamètre unique pour toute la conduite.

Cette formule est à employer de préférence lors du premier calcul de T. En effet, il est absolument superflu de calculer une première valeur de $T$ avec toutes les complicalions que comporle l'adoption d'une conduite a plusieurs diamètres. On ne passera à celle-ci, en tant que la nécessité se présentera, que lorsque lil valeur de $\mathrm{T}$ será élablie d'une manière approximativement correcte.

En raison de ce qui précède, l'importance de celte formule

$$
\mathrm{D}=\sqrt[7]{\frac{\mathrm{TL}}{y\left(\mathrm{~L}-l_{0}\right)+h_{0} l_{0}}}
$$

est tout à fait évidente : c'est la formule à employer toutes les fois où l'on peut se contenter d'une conduite d'un seul diamètre constant. Or, celui-ci impliquant la valeur minimum de la vitesse d'eau U, l'on fera toujours bien de commencer par l'emploi de cetle formule et ne passer à $n=1,2 \ldots$. etc., que lorsque la vitesse maximum de l'eau le permet. 